\title{
SPACES OF HOLOMORPHIC FUNCTIONS AND HILBERT-SCHMIDT SUBSPACES
}

\author{
by EBERHARD GERLACH*
}

(Received 15 June, 1970)

In this note we construct certain Hilbert subspaces with Hilbert-Schmidt imbedding, for an arbitrary proper functional Hilbert space which consists of holomorphic functions. This work extends results of Chapter III in [1] and has applications in the regularity problem for generalised eigenfunctions (in particular to Theorem 2 in [2]). For an exposition of reproducing kernels and Bergman's kernel function we refer to [4].

Let $P$ denote a polydisc $P=\left\{z \in \mathrm{C}:\left|z_{j}-a_{j}\right|<r_{j}(j=1, \ldots, n)\right\}$ and $\mathscr{B}=\mathscr{B}(P)$ the space of all functions that are holomorphic in $P$ and square integrable over $P$ with respect to Lebesgue measure on $\mathbf{R}^{2 n}$. $\mathscr{B}$, endowed with the $L^{2}$-norm, has a reproducing kernel $B(z, \zeta)$ which is Bergman's kernel; the function $B: P \rightarrow \mathscr{B}$ given by $z \mapsto B(\cdot, z)$ is (strongly) conjugateholomorphic (i.e. $\bar{z} \mapsto B(\cdot, z)$ is holomorphic from $\bar{P}$ to $\mathscr{B}$ ). The following result extends Theorem III.1 of [1] (which corresponds to the case $\alpha=1$ below).

THEOREM 1. There is a positive selfadjoint operator T of Hilbert-Schmidt type in the space $\mathscr{B}$ with Bergman's kernel $B(z, \zeta)$, with the following properties. For every $\alpha \geqq 0$, the Hilbert subspace $T^{\alpha} \mathscr{B} \equiv \mathscr{B}_{\alpha}$ of $\mathscr{B}$ with norm $\|\varphi\|_{\alpha}=\left\|T^{-\alpha} \varphi\right\|_{\mathscr{B}}$ contains all functions $B(\cdot, \zeta)(\zeta \in P)$, and so does the nuclear countably-Hilbert space $\mathscr{B}_{\infty} \equiv \bigcap_{\alpha \in \mathbf{R}} \mathscr{B}_{\alpha}=\bigcap_{k=0}^{\infty} \mathscr{B}_{k}$

$$
\left(\text { with metric } \rho(0, u)=\sum_{k=0}^{\infty} 2^{-k} \frac{\|u\|_{k}}{1+\|u\|_{k}}\right)
$$

moreover the mapping $\zeta \mapsto B(\cdot, \zeta)$ is conjugate-holomorphic in the norm of every $\mathscr{B}_{a}$ and in the metric of $\mathscr{B}_{\infty}$.

Proof. We construct the operator $T$. Let $m$ denote a multi-index $\left(m_{1}, \ldots, m_{n}\right)$ of positive integers, and write $\prod_{j=1}^{n} m_{j}=\bar{m}$. The monomials $\chi_{m}(z)=\prod_{j=1}^{n}\left(z_{j}-a_{j}\right)^{m_{j}-1}$ are orthogonal in $\mathscr{B}$; their norms are $\left\|\chi_{m}\right\|=\pi^{\frac{1}{2} n} \bar{m}^{-\frac{1}{2}} r_{1}^{m_{1}} \ldots r_{n}^{m_{n}}$. Set $\varphi_{m}=\left\|\chi_{m}\right\|^{-1} \chi_{m}$; the $\varphi_{m}$ form a complete orthonormal system ("CONS") for $\mathscr{B}$. The reproducing kernel $B(z, \zeta)$ is represented by $B(\cdot, \zeta)=\sum_{m} \overline{\varphi_{m}(\zeta)} \varphi_{m}$, the series converging in the norm of $\mathscr{B}$. We define the operator $T$ by

$$
T \varphi_{m}=\bar{m}^{-1} \varphi_{m}
$$

$T$ is Hilbert-Schmidt, since $\sum\left\|T \varphi_{m}\right\|^{2}=\sum \bar{m}^{-2}<\infty$. For every real number $\alpha$ and every A3014.

* This research was supported in part by the National Research Council of Canada, Operating Grant 
$\zeta \in P$ we have

$$
\sum_{m}\left|\bar{m}^{\alpha} \varphi_{m}(\zeta)\right|^{2}<\infty
$$

and consequently

$$
B(\cdot, \zeta)=\sum\left[\bar{m}^{\alpha} \overline{\varphi_{m}(\zeta)}\right]\left[T^{\alpha} \varphi_{m}\right] \in T^{\alpha} \mathscr{B}=\mathscr{B}_{\alpha} \text { for } \alpha \geqq 0 .
$$

Since each $\mathscr{B}_{\alpha}$ is dense in $\mathscr{B}_{\beta}$ for any $0 \leqq \beta \leqq \alpha$, we may extend the chain of spaces $\mathscr{B}_{\alpha}(\alpha \geqq 0)$ by duality. For every $\alpha>0$, regard $\mathscr{B}$ as a dense subspace of the anti-dual $\left(\mathscr{B}_{\alpha}\right)^{*}$, the imbedding given by

$$
f(\varphi)=(f, \varphi)_{0} \quad \text { for every } \varphi \in \mathscr{B}_{\alpha} \text { and } f \in \mathscr{B}=\mathscr{B}_{0} .
$$

Setting $\left(\mathscr{B}_{\alpha}\right)^{*}=\mathscr{B}_{-\alpha}$, we thus obtain a continuous scale of Hilbert spaces in the sense of S. G. Kreĭn and others (cf. [3] for instance), and this scale is nuclear, since the imbedding $\mathscr{B}_{\alpha} \subset \mathscr{B}_{\beta}$ is nuclear whenever $\alpha-\beta>2$. In this chain we can define any (real) power of $T$ by $T^{\beta} \varphi_{m}=\bar{m}^{-\beta} \varphi_{m}$ for arbitrary real $\beta$, and then $T^{\beta} \mathscr{B}=\mathscr{B}_{\beta}$. Moreover, all the spaces $\mathscr{B}_{\beta}$ consist of holomorphic functions on $P$, and the space $\mathscr{B}_{\beta}$ has a reproducing kernel given by $B_{\beta}(\cdot, \zeta)=T^{2 \beta} B(\cdot, \zeta)(\beta \in \mathbf{R})$. Also note that, for all $\alpha, \beta \in \mathbf{R}$, we have

$$
T^{\beta} B(\cdot, \zeta) \in \mathscr{B}_{\alpha} \text { for every } \zeta \in P \text {. }
$$

At the very end of [1] it was proved by direct calculation that $z \mapsto B(\cdot, z)$, regarded as a function from $P$ into $\mathscr{B}_{1}$, is (strongly) conjugate holomorphic, i.e., it was shown that

$$
\partial B(\cdot ; \zeta) / \partial \bar{\zeta}_{j}=\lim _{h \rightarrow 0} \hbar^{-1}\left[B\left(\cdot, \zeta+h \varepsilon_{j}\right)-B(\cdot, \zeta)\right]
$$

exists in the norm of $\mathscr{B}_{1}$. (Note that the derivative on the left can always be defined pointwise by

$$
\frac{\partial B(\cdot, \zeta)}{\partial \bar{\zeta}_{j}}(z)=\frac{\partial B(z, \zeta)}{\partial \bar{\zeta}_{j}}
$$

which is known to exist.) In a completely analogous way one may calculate that, for any real numbers $\alpha$ and $\beta$, the function $B_{\beta}: P \rightarrow \mathscr{B}_{\alpha}$ given by $z \mapsto B_{\beta}(\cdot, z)$ is conjugate-holomorphic in the norm of $\mathscr{B}_{\alpha}$.

Finally, set $\mathscr{B}_{\infty}=\bigcap_{\alpha \in \mathbf{R}} \mathscr{B}_{\alpha}$ and $\mathscr{B}_{-\infty}=\bigcup_{\alpha \in \mathbf{R}} \mathscr{B}_{\alpha}$, wit hthe corresponding projective and inductive limit topology, respectively. $\mathscr{B}_{\infty}$ is also equal to the nuclear countably-Hilbert space $\bigcap_{k \geqq 0} \mathscr{B}_{k}$ ( $k$ an integer); it is a Fréchet space in the metric (" quasi-norm ")

$$
\sum 2^{-k} \frac{\|u\|_{k}}{1+\|u\|_{k}}
$$

Then $z \mapsto B_{\beta}(\cdot, z)$ (for every fixed $\beta \in \mathbf{R}$ ) is also conjugate-holomorphic as a function from $P$ into $\mathscr{B}_{\infty}$. The nuclear space $\mathscr{B}_{-\infty}$ is the strong anti-dual of $\mathscr{B}_{\infty}$ (and vice-versa), and it is 
continuously imbedded in the space $H(P)$ of all holomorphic functions in $P$ with the topology of uniform convergence on compacts. The proof is complete.

Remark. The space $\mathscr{B}_{\beta}$ has reproducing kernel $T^{2 \beta} B(\cdot, \zeta)$. In any proper functional Hilbert space $\mathscr{H}$ on a set $E$, with reproducing kernel $K$, all bounded and certain unbounded operators $L$ have a representation of the following kind:

$$
(L f)(x)=(L f, K(\cdot, x))=\left(f, L^{*} K(\cdot, x)\right) \text { for } x \in E,
$$

i.e.,

$$
(L f)(x)=(f, \Lambda(\cdot, x)),
$$

where $\Lambda(\cdot, x)=L^{*} K(\cdot, x)$ is the " kernel of $L$ ". In the present situation, for any $\alpha, \beta \in \mathbf{R}$, the operator $T^{\alpha}$ in $\mathscr{B}_{\beta}$ is represented by a kernel $S_{\alpha, \beta}$, say, namely

$$
\begin{aligned}
& S_{\alpha, \beta}(\cdot, z)=T^{\alpha} B_{\beta}(\cdot, z)=T^{\alpha+2 \beta} B(z)=\sum \bar{m}^{-(\alpha+2 \beta)} \overline{\varphi_{m}(\zeta)} \varphi_{m} ; \\
& \left(T^{\alpha} \varphi\right)(z)=\left(\varphi, T^{\alpha} B_{\beta}(\cdot, z)\right)_{\beta} .
\end{aligned}
$$

(Note that all $\varphi_{m}$ lie in $\mathscr{B}_{\infty}$.)

We now turn to the general case which corresponds to the preceding theorem. Let $D$ be a connected domain in $\mathbf{C}^{n}$, and $\{\mathscr{F}, D\}$ an arbitrary proper functional Hilbert space consisting of functions that are holomorphic in $D$; denote the reproducing kernel of $\mathscr{F}$ by $K(z, \zeta)$. Let $P$ be any polydisc whose closure is contained in $D$. Then we have the following result.

THEOREM 2. There exists a Hilbert subspace $\Phi$ of $\mathscr{F}$ containing all functions $K(\cdot, \zeta)(\zeta \in P)$, such that the imbedding of $\Phi$ into $\mathscr{F}$ is Hilbert-Schmidt, and the function $P \rightarrow \Phi$ given by $\zeta \mapsto K(\cdot, \zeta)$ is conjugate-holomorphic in the norm of $\Phi$.

Proof. Let $\left.\mathscr{F}\right|_{P}$ be the space of restrictions to $P$ of functions in $\mathscr{F}$. Since $D$ is connected and the functions in $\mathscr{F}$ are holomorphic, the subspace $N(P)$ of $\mathscr{F}$ of functions vanishing identically on $P$ is zero. Thus $\left.\mathscr{F}\right|_{P}$ is "the same" as $\mathscr{F} \ominus N(P)=\mathscr{F}$. All functions in $\left.\mathscr{F}\right|_{P}$ are holomorphic in a neighbourhood of the closure of $P$, hence square integrable on $P$, and so $\left.\mathscr{F}\right|_{P}(=\mathscr{F})$ is a Hilbert subspace of $\mathscr{B}(P)=\mathscr{B}$ with Bergman kernel $B$. Let $G$ be the “ kernel of $\mathscr{F}$ in $\mathscr{B}$ ", i.e., $(f, h)_{\mathscr{A}}=(f, G h)_{\mathscr{F}}$ for $f \in \mathscr{F}, h \in \mathscr{B}$; its square root (taken in $\left.\mathscr{B}\right)$ is the canonical partial isometry of $\mathscr{B}$ onto $\mathscr{F}$. Then the reproducing kernel of $\left.\mathscr{F}\right|_{P}$ is the restriction of $K(z, \zeta)$ to $P \times P$, and $\left.K(\cdot, \zeta)\right|_{P}=G B(\cdot, \zeta)$ for $\zeta \in P$. Let $\left\{\varphi_{m}\right\}$ and $T$ in $\mathscr{B}$ be as described in Theorem 1. Then

$$
\left.K(\cdot, \zeta)\right|_{P}=G B(\cdot, \zeta)=\sum_{m} \overline{m^{\alpha}} \overline{\varphi_{m}(\zeta)} G T^{\alpha} \varphi_{m}
$$

for all $\alpha \geqq 0$, and

$$
\sum_{m}\left\|G T^{\alpha} \varphi_{m}\right\|_{\mathscr{F}}^{2}=\sum_{m}\left\|G^{\frac{1}{2}} T^{\alpha} \varphi_{m}\right\|_{\mathscr{B}}^{2} \leqq\|G\|_{\mathscr{B}} \sum_{m}\left\|T^{\alpha} \varphi_{m}\right\|_{\mathscr{B}}^{2} \quad\left(\left\|G^{\frac{1}{2}}\right\|^{2}=\|G\| \text { in } \mathscr{B}\right) .
$$

Now let $\left\{\psi_{m}\right\}$, where $m$ varies over all multi-indices of positive integers, be a CONS in 
$\mathscr{F}=\left.\mathscr{F}\right|_{\boldsymbol{P}}$, and put

$$
L \psi_{m}=G T^{\alpha} \varphi_{m}=\bar{m}^{-\alpha} G \varphi_{m}
$$

for some fixed $\alpha$. Then $K(\cdot, \zeta) \in L \mathscr{F}$ for all $\zeta \in P$, and, if $\alpha \geqq 1$, then $L$ is Hilbert-Schmidt in $\mathscr{F}$.

Let $\alpha \geqq 1$ (fixed) from now on, and make $L \mathscr{F}$ into a Hilbert-Schmidt subspace $\Phi$ of $\mathscr{F}$ by defining the following norm on it:

$$
\|v\|_{\Phi}^{2}=\inf \left\{\sum\left|\xi_{m}\right|^{2}: v=\sum \xi_{m} L \psi_{m} \text { in } \mathscr{F}\right\} .
$$

We check that $K(\cdot, \zeta)$ is conjugate-holomorphic in the norm of $\Phi$. It is known that the two limits

$$
\partial K(z, \zeta) / \partial \zeta_{j}=\lim _{h \rightarrow 0} \hbar^{-1}\left(K(\cdot, z), K\left(\cdot, \zeta+h \varepsilon_{j}\right)-K(\cdot, \zeta)\right)_{\mathscr{F}}
$$

and

$$
\lim _{h \rightarrow 0} h^{-1}\left[K\left(\cdot, \zeta+h \varepsilon_{j}\right)-K(\cdot, \zeta)\right] \text { in the norm of } \mathscr{F}
$$

exist, and the value of the second of these at $z$ is just the first limit. Write

$$
h^{-1}\left[K\left(\cdot, \zeta+h \varepsilon_{j}\right)-K(\cdot, \zeta)\right]=K_{\zeta, h} .
$$

Because of the uniqueness of limits we only have to show now that the $K_{\zeta, h}$ converge, as $h \rightarrow 0$, in the norm of $\Phi$; then their limit must lie in $\Phi$ and equal $\partial K(z, \zeta) / \partial \zeta_{j}$. If $\zeta \in P$, then

$$
K(\cdot, \zeta)=\sum_{m} \overline{\bar{m}^{\alpha}} \overline{\varphi_{m}(\zeta)} L \psi_{m}
$$

and

$$
\|K(\cdot, \zeta)\|_{\Phi}^{2} \leqq \sum_{m}\left|\bar{m}^{\alpha} \varphi_{m}(\zeta)\right|^{2} .
$$

Due to our constructions, $G$ restricted to $\mathscr{B}_{\alpha}$ is a bounded operator of $\mathscr{B}_{\alpha}$ into $\Phi$; moreover $K_{\zeta, h}=G h^{-1}\left[B_{\alpha}\left(\cdot, \zeta+h \varepsilon_{j}\right)-B_{\alpha}(\cdot, \zeta)\right] \equiv G B_{\alpha, \zeta, h}$, and the $B_{\alpha, \zeta, h}$ converge in $\mathscr{B}_{\alpha}$ by Theorem 1 . Thus the $K_{\zeta, h}$ converge in the norm of $\Phi$ as $h \rightarrow 0$. The proof is complete.

Remark. One could cover $D$ by a sequence of polydiscs $P_{k}$ whose closures all lie in $D$, redefine $L$ and $\Phi$ suitably, and obtain the conclusions of Theorem 2 simultaneously for all $\zeta \in D$.

It would be desirable to obtain a slightly better result than the space $\Phi$ in Theorem 2 , namely a nuclear Fréchet space $\Psi$ continuously imbedded in $\mathscr{F}$, containing all $K(\cdot, \zeta)(\zeta \in D)$, such that the map $D \rightarrow \Psi$ given by $\zeta \mapsto K(\cdot, \zeta)$ is conjugate holomorphic in the topology of $\Psi$. For the present we leave aside the problem of constructing such a nuclear subspace in a direct way (without recourse to the space $\mathscr{B}(P)$ ).

If we restrict ourselves to a fixed polydisc whose closure lies in $D$, and if the closure $\mathscr{M}$ of $\left.\mathscr{F}\right|_{P}=\mathscr{F}$ in $\mathscr{B}=\mathscr{B}(P)$ reduces the operator $T$, we can proceed as follows. Let $Q$ be the 
orthogonal projection onto $\mathscr{M}$ in $\mathscr{B}$. Then $Q$ commutes with every $T^{\alpha}$, and $Q T$ generates the nuclear Hilbert scale $\left\{Q \mathscr{B}_{\alpha}: \alpha \in \mathbf{R}\right\}, Q \mathscr{B}_{\alpha}=Q T^{\alpha} \mathscr{B}$. In this case the nullspace of the kernel $G$ ( $G$ defined in the proof of Theorem 2) equals the orthogonal complement of $\mathscr{M}$, and, as $G$ is injective on every $Q \mathscr{B}_{\alpha}$, we simply transfer the norm \|\|$_{\alpha}$ from $Q \mathscr{B}_{\alpha}$ to $G Q \mathscr{B}_{\alpha}=G \mathscr{B}_{\alpha}$ and obtain the nuclear Fréchet space $\Psi=\bigcap\left\{G \mathscr{B}_{\alpha},\|\|_{\alpha}\right\}(\alpha \in \mathbf{R})$, which contains all $K(\cdot, \zeta)(\zeta \in P)$. The norm \|\|$_{\alpha}$ on $G \mathscr{B}_{\alpha}$ is the same as that given in the above definition of a norm on $\Phi$ (when we used $\left.L \psi_{m}=\bar{m}^{-\alpha} G \varphi_{m}\right)$. Since $\zeta \mapsto K(\cdot, \zeta)$ is conjugate-holomorphic in every norm \|\|$_{\alpha}$, it is conjugate-holomorphic in the metric

$$
\sum 2^{-k} \frac{\|u\|_{k}}{1+\|u\|_{k}} \text { of } \Psi .
$$

REMARK. If $\Phi^{*}$ and $\Psi^{*}$ are the (strong) anti-duals of the $\Phi$ and $\Psi$ above, then $\mathscr{F} \subset \Phi^{*} \subset H(D)$ or $\mathscr{F} \subset \Psi^{*} \subset H(D)$ with continuous linear imbeddings, where $H(D)$ is the space of all holomorphic functions on $D$ with the topology of uniform convergence on compacts.

\section{REFERENCES}

1. E. Gerlach, On spectral representation for selfadjoint operators. Expansion in generalized eigenelements, Ann. Inst. Fourier Grenoble, 15 (1965), 537-574.

2. E. Gerlach, Mean value properties of generalised eigenfunctions, Proc. Edinburgh Math. Soc. (2) 17 (1970), 155-158.

3. S. G. Krê̌n and Ju. I. Petunin, Scales of Banach spaces (Russian), Uspehi Mat. Nauk 21 (1966), no. 2 (128), 89-168. [English translation in Russian Math. Surveys 21 (1966), no. 2, 85-159.]

4. H. Meschkowski, Hilbertsche Räume mit Kernfunktion (Berlin, 1962).

\section{UNIVERSITY OF BRITISH COLUMBIA}

VANCOUyer 8, B.C., Canada

and

UNIVERSITY OF EDINBURGH 